\title{
Open-String Gromov-Witten Invariants: Calculations and a Mirror "Theorem"
}

\author{
Tom Graber and Eric Zaslow
}

October 26, 2018

\begin{abstract}
We propose localization techniques for computing Gromov-Witten invariants of maps from Riemann surfaces with boundaries into a CalabiYau, with the boundaries mapped to a Lagrangian submanifold. The computations can be expressed in terms of Gromov-Witten invariants of one-pointed maps. In genus zero, an equivariant version of the mirror theorem allows us to write down a hypergeometric series, which together with a mirror map allows one to compute the invariants to all orders, similar to the closed string model or the physics approach via mirror symmetry. In the noncompact example where the Calabi-Yau is $K_{\mathbb{P}^{2}}$, our results agree with physics predictions at genus zero obtained using mirror symmetry for open strings. At higher genera, our results satisfy strong integrality checks conjectured from physics.
\end{abstract}

\section{Introduction}

\subsection{The Physics}

Mirror symmetry is famous for being able to predict Gromov-Witten invariants of Calabi-Yau manifolds. The basic conjecture is that there is a duality between string theories on mirror Calabi-Yau manifolds. As a consequence, the topological field theory defined from the A-twist of one Calabi-Yau manifold is equal to the topological B-twist of the mirror. Both twists can be performed on Calabi-Yau target manifolds. From a practical point of view, in order to obtain enumerative predictions, one needs to know the theory on the B-model (in this case, defined through classical period integrals) as well as an identification of the parameter spaces for both theories - the "mirror map." To extract integer-valued invariants, one needs an all-genus "multiple-cover" formula. The technology for finding mirror manifolds [3] 
and performing calculations of the mirror map [19] is well established. The multiple-cover formula was found at genus zero in [6] and generalized to all genera in [13]. At higher genus, the BCOV equations [4] can be used (up to an ambiguity) to calculate Gromov-Witten invariants. Mathematical verification of these predictions has only be performed in the noncompact setting [17] 22]. The integer invariants are known as Gopakumar-Vafa invariants, and still await a mathematical definition.?

String theory in a spacetime-filling brane is described by maps from Riemann surfaces in which the boundaries are mapped to submanifolds in the Calabi-Yau. Other data such as gauge fields on the submanifolds may be present. Not all submanifolds preserve the supersymmetries necessary to perform the topological twisting. Lagrangian submanifolds preserve the A-twist, while holomorphic submanifolds preserve the B-twist. The mirror symmetry duality implies that to each A-brane there corresponds a mirror B-brane, so that the resulting string theories are still equivalent. As a consequence, the resulting topological field theories must also be the same. On the B-model side, this resulting theory is known [4] [30] to be given by a holomorphic version of Chern-Simons theory.

The insight of Aganagic and Vafa [1] was an identification of special pairs of mirror branes in the setting where one Calabi-Yau was a noncompact toric variety, as well as dimensional reduction of the holomorphic Chern-Simons theory. Those authors chose a Lagrangian A-brane whose corresponding B-brane was a holomorphic curve inside the mirror. (Such pairs were also described in [16].) Though holomorphic Chern-Simons theory is defined for complex three-folds, the reduction to complex curves can be performed, and results in a computable integral of a meromorphic two-form. Aganagic and Vafa then found a mirror map identifying brane moduli, so that the expansion of a superpotential in the mirror coordinates encoded the (conjectural) Gromov-Witten invariants involving holomorphic maps from Riemann surfaces with boundaries to the noncompact Calabi-Yau, where the boundaries get mapped to the relevant Lagrangian submanifold. What resulted was a prediction of these would-be open-string Gromov-Witten invariants at genus zero - i.e., for maps from disks. Such invariants have not yet been defined rigorously, from a mathematical point of view.

The multiple-cover formula for open-string Gromov-Witten invariants was found in [29]. This has been generalized to Riemann surfaces with

\footnotetext{
${ }^{1}$ See [5], [20], and [18] for progress in this direction.

${ }^{2}$ Insofar as these invariants are related to Fukaya-Floer theory, much of the work towards making a rigorous mathematical theory of them has been carried out in [10].
} 
more than one boundary component in 24]. These formulas have been verified through localization calculations in [25] and 21]. Recently, Aganagic, Klemm and Vafa [2] expanded this mirror procedure ("B-model") in a number of new examples, and were able to make integer predictions of the invariants. Mariño and Vafa [27] have done the same for higher-genus, multiple-boundary invariants using Chern-Simons duals. In this note we will perform localization calculations to provide an explicit "A-model" verification of these predictions, and use an equivariant mirror theorem 8 to compute. The results we obtain match those authors' perfectly, including a dependence on an additional $\mathbb{Z}$-valued parameter.

In addition, we are able to extend our calculations to higher genus Riemann surfaces with boundary, and to multiple boundary components. No physical predictions have been made of these numbers, though there are strong integrality properties predicted through the work of Ooguri and Vafa [29] and of Labastida, Mariño and Vafa 24]. These requirements have been met in all compuatations checked.

\subsection{The Math}

In 23], Kontsevich defines a moduli space of stable maps, which allows one to compute Gromov-Witten invariants in many (toric) examples through localization techniques. The basic idea of these calculations is straightforward. While the moduli space of maps is quite complicated, the subspace which is fixed under the natural torus action is simple. By applying a localization theorem we can turn integrals over the entire space of maps into integrals over the fixed locus which we can compute.

Kontsevich used this method to compute genus zero Gromov-Witten invariants of a homogeneous space. In this case, the space of maps is smooth, and the Bott residue theorem suffices to reduce the computation of the invariants to integrals over $\bar{M}_{0, n}$.

For higher genus invariants, or nonconvex target spaces, a virtual localization theorem is needed, since the Gromov-Witten invariants are defined to be integrals against a virtual fundamental class. In [15], such a theorem is proven, provided the target space is a smooth algebraic variety with an algebraic $\mathbb{C}^{*}$ action.

The current setting, however, is non-algebraic. Moreover, there is not (yet) a mathematical formulation of the Gromov-Witten invariants associated to maps from Riemann surfaces with boundary. One then hopes to define a virtual fundamental class and prove a localization theorem for this class in a symplectic setting. We do not prove such a theorem in this pa- 
per. Instead, we perform a calculation in the spirit of localization, and find agreement with the predictions of [2].

We now briefly describe the calculation. The Calabi-Yau we consider is $K_{\mathbb{P}^{2}}$, the total space of the canonical bundle of $\mathbb{P}^{2}$, and the Lagrangian submanifold $L$ is constructed from the co-normal bundle over a straight line in the toric polytope (the image of the moment map used in the construction of $K_{\mathbb{P}^{2}}$ ). It intersects the $\mathbb{P}^{2}$ in an $S^{1}$ which we label $S_{L}^{1}$. This Lagrangian is preserved under a real torus, which acts on the moduli space of maps. The fixed points correspond to source curves which map equivariantly to invariant curves in the image. The component of such a source curve containing the (single) connected boundary component must map to a disk in $\mathbb{P}^{2}$ ending on $S_{L}^{1}$, and is determined by the sign of the winding of the loop around $S_{L}^{1}$. In this way, the fixed-locus components should be described by the graphs that Kontsevich introduced, with a single "leg" attached corresponding to the boundary disk. To each graph $\Gamma$ is associated a moduli space $M_{\Gamma}$ which is a product over vertices $v$ of $\mathcal{M}_{\text {genus }(v) \text {,valence }(v) \text {, i.e. the }}$ moduli of the contracted components. The integrals are performed using Faber's algorithm.

The alert reader may note that the leg is specified by an attachment location and a winding parameter. Indeed, we define a winding-dependent integral involving stable maps with one marked point, which, together with a winding-dependent multiplicative constant, yields the same result. This observation, together with an equivariant mirror theorem, can be used to write a hypergeometric series and mirror map which computes all genus-zero open-string Gromov-Witten invariants.

In the next section, we describe more carefully the geometric setting which will serve as the main example of the localization formulas obtained in Section 3. Genus-zero invariants are computed with the equivariant mirror theorem in Section 4 , and a comparison with the physics open mirror symmetry procedure is made. We conclude with some example calculations involving integer invariants (BPS numbers), and some comments about future directions.

\section{Defining Terms}

Define the "symplectic quotient"

$$
M \equiv\left\{\left|z_{1}\right|^{2}+\left|z_{2}\right|^{2}+\left|z_{3}\right|^{2}-3\left|z_{4}\right|^{2}=r\right\} / S^{1},
$$


where $r>0$ and $S^{1}$ acts by $\theta:\left(z_{1}, \ldots, z_{4}\right) \mapsto\left(e^{i \theta} z_{1}, e^{i \theta} z_{2}, e^{i \theta} z_{3}, e^{-3 i \theta} z_{4}\right)$, i.e. with weights $(1,1,1,-3)$. Then $M \cong K_{\mathbb{P}^{2}}$, the total space of the canonical bundle of $\mathbb{P}^{2}$. Note that $z_{4}$ parametrizes the fiber, and that $z_{1}, z_{2}$, and $z_{3}$ cannot be simultaneously zero.

In the patch $U \equiv\left\{z_{3} \neq 0\right\}$, we can parametrize $M$ with three coordinates $z, n$, and $f$ defined by

$$
z=a z_{1} / z_{3}, n=z_{2} / z_{3}, f=z_{4} z_{3}^{3},
$$

where the constant $a=\sqrt{(r+c) /(r-c)}$ is included for later convenience $(c<r$ is defined in $\operatorname{Eq}(2))$. Note that $z, n, f$ determine $z_{1}, \ldots, z_{4}$ via Eq.(1), up to the quotiented $S^{1}$ action.

There is a residual $\left(S^{1}\right)^{3}$ action on $M$. We will exploit this later to perform localization calculations. In fact, it is often enough to consider a generic subgroup $S^{1} \subset\left(S^{1}\right)^{3}$, which we define by weights $\lambda_{z}, \lambda_{n}, \lambda_{f}$. Explicitly, $\theta:(z, n, f) \mapsto\left(e^{i \lambda_{z} \theta} z, e^{i \lambda_{n} \theta} n, e^{i \lambda_{f} \theta} f\right)$.

The symplectic form $\omega=\frac{1}{2} \sum_{i=1}^{4} d\left|z_{i}\right|^{2} \wedge d \theta_{i}$ descends to $M$, and we define the Lagrangian submanifold $L$ by

$$
\begin{array}{r}
\left|z_{1}\right|^{2}-\left|z_{3}\right|^{2}=c, \\
\left|z_{2}\right|^{2}-\left|z_{4}\right|^{2}=0, \\
\operatorname{Arg}\left(z_{1} z_{2} z_{3} z_{4}\right)=0,
\end{array}
$$

where $c<r$. Note that these equations make sense on $M$. The $S^{1}$ action doesn't affect the absolute values, but in order to preserve $L$, we must require that

$$
\lambda_{z}+\lambda_{n}+\lambda_{f}=0
$$

In terms of weights $\lambda_{i}$ for the $z_{i}$, we can write $\lambda_{z}=\lambda_{1}-\lambda_{3}, \lambda_{n}=\lambda_{2}-\lambda_{3}$, and $\lambda_{f}=-\left(\lambda_{n}+\lambda_{z}\right)=2 \lambda_{3}-\lambda_{1}-\lambda_{2}$. Note that $L$ is obtained by a co-normal construction of a linear subspace of the image of the moment maps $\left|z_{i}\right|^{2} / 2$.

Define $\mathbb{P}^{2}$ by $z_{4}=0$ and note that $L \cap \mathbb{P}^{2}$ is a circle $S_{L}^{1}$ defined by $f=$ $n=0,|z|=1$. Noting that $L$ has the topology of $S^{1} \times \mathbb{R}^{2}$, we coordinatize $L$ with a real angular variable $\theta$ and a complex variable $x$ (this is not meant to suggest that $L$ has a complex structure). Let us define $\varphi: L \hookrightarrow M$ in the coordinates $\left(z_{1}, \ldots, z_{4}\right)$ by

$$
\varphi(\theta, x)=\left(\sqrt{|x|^{2}+\frac{r+c}{2}} e^{i \theta} e^{i \theta_{3}}, x e^{i \theta_{3}}, \sqrt{|x|^{2}+\frac{r-c}{2}} e^{i \theta_{3}}, \bar{x} e^{-i \theta} e^{-3 i \theta_{3}}\right) .
$$


Note that $\theta_{3}$ parametrizes the $S^{1}$ ambiguity, whereas the values of $z, n, f$ are well-defined. Note also that $S_{L}^{1}$ is the image of $S^{1} \times(0,0) \subset S^{1} \times \mathbb{R}^{2}=L$

One calculates that along $S_{L}^{1}$,

$$
\begin{array}{r}
\varphi_{*}\left(\frac{\partial}{\partial \theta}\right)=\frac{\partial}{\partial \theta_{z}}=i z \frac{\partial}{\partial z}-i \bar{z} \frac{\partial}{\partial \bar{z}} \\
\varphi_{*}\left(\frac{\partial}{\partial x}\right)=\frac{\partial}{\partial n}+e^{-i \theta_{z}} \frac{\partial}{\partial \bar{f}}
\end{array}
$$

We will be interested in holomorphic maps from a disk into $M$, such that the boundary of the disk is mapped into $L$. The infinitesimal deformation of such a map is a holomorphic section of the pull-back of $T M$ which lives in the pull back of $T L \subset T M$ along the boundary. Therefore, we now describe how $T L$ sits inside $T M$. In fact, we will only need to describe $\left.\left.T L\right|_{S_{L}^{1}} \subset T M\right|_{S_{L}^{1}}$ since the boundary of the disk will land inside $S_{L}^{1}$ via a holomorphic map. As $S_{L}^{1}$ is contained in our patch $U$, we can identify $T M$ with the trivial bundle $\mathbb{C}^{3}$ generated by $\frac{\partial}{\partial z}, \frac{\partial}{\partial n}, \frac{\partial}{\partial f}$.

The holomorphic map of most interest to us will be

$$
\psi_{w}: D \rightarrow M
$$

where $D$ is the unit disk $\{|u| \leq 1\}$ and $\psi_{w}(u)=\left(z=u^{w}, n=0, f=0\right)$. Note that $\psi_{w}: \partial D \rightarrow S_{L}^{1}$ has winding number $w$. (A similar map based at an opposite pole of an invariant $\mathbb{P}^{1}$ can be defined when $w<0$.) When we pull back $T M$ to $D$ via $\psi_{w}$, the totally real sub-bundle $T L \subset T M$ can be described by the real span of a basis defined by a unitary matrix acting on the frame $\frac{\partial}{\partial z}, \frac{\partial}{\partial n}, \frac{\partial}{\partial f}$. Let $\theta$ parametrize $\partial D$. Then over the point $u=e^{i \theta} \in \partial D$, we see from $\mathrm{Eq}(4)$ that $T L$ is defined by the matrix

$$
A=\frac{1}{\sqrt{2}}\left(\begin{array}{ccc}
i \sqrt{2} e^{i w \theta} & 0 & 0 \\
0 & 1 & i \\
0 & e^{-i w \theta} & -i e^{-i w \theta}
\end{array}\right) .
$$

Note that this matrix is unitary, and its determinant is constant - a consequence of the special Lagrangian condition that the phase of the holomorphic three-form is constant along $L$.

\section{Localization Calculation}

The authors of both [21] and 25] find an algebraic moduli space in which the space of stable maps from Riemann surfaces with boundary embeds. 
One hopes to be able to approach the general non-algebraic problem via a virtual localization formula (not proven) for this space. In the following, we assume some such formula is valid (see Eq.(可)). In fact, our calculations will ultimately be torus weight-dependent, producing a discrete dependence of the answers which matches physical predictions. The origins of this dependence are unclear to us. However, the actual calculation is straightforward. As in the closed string case, while the global geometry of the moduli space of maps is complicated, the geometry of the fixed locus is simple. Indeed, the fixed loci are identical to those that arise in space of maps from closed Riemann surfaces. (We exploit this observation in Section 1 .) Moreover, the deformation theory that governs the contribution of such a fixed locus to the integral is essentially the same as in the closed case. As a result, even in the absence of a general theory to justify such a computation, we have a natural method for finding these invariants.

Let us describe the localization calculation. As in [23], the fixed-point locus will be described by decorated graphs which relate: the invariant $\mathbb{P}^{1}$ 's where genus-zero components of the source curves are mapped; the fixed points in $\mathbb{P}^{2}$ where contracted components are mapped; and the disks sitting inside invariant $\mathbb{P}^{1}$ 's where disk components are mapped. We therefore define a decorated graph $\Gamma=(\mathcal{V}, \mathcal{E}, \mathcal{L})$ as triple of vertices $\mathcal{V}$, edges $\mathcal{E}$, and "legs" $\mathcal{L}$ defined as follows. Each vertex $v \in \mathcal{V}$ carries a label $i(v)$ of a torus fixed point among $p_{1}, \ldots, p_{3}$ in $\mathbb{P}^{2}$ (here $p_{i}$ is the point in $\mathbb{P}^{2}$ with $z_{i}$ as the only non-zero coordinate), as well as the genus $g(v)$ of a contracted component $C_{v}$ of the source curve. Each edge $e \in \mathcal{E}$ carries a positive degree label $d_{e}$ of the map from the edge component $C_{e}$, and an unordered pair $(i(e), j(e))$ of vertices that the edge joins. A "leg" $l \in \mathcal{L}$ will represent a disk source component $C_{l}$ with boundary landing in $L \cap \mathbb{P}^{2}$, i.e., $S_{L}^{1}$ in the example in this paper. Each leg is labelled by a winding $w(l)$ describing the degree of the map from $\partial C_{l}$ to $S_{L}^{1}$. In addition, we may wish to refer to $i(l)$, a label of the fixed point that the center of the disk $C_{l}$ is mapped to, and $j(l)$, the fixed point at the other pole of the invariant $\mathbb{P}^{1} \subset \mathbb{P}^{2}$ containing the image of $C_{l}$. In the example of this paper, positive winding will demand that $i(l)=p_{3}$ while negative winding demands $i(l)=p_{1}$. The valence of a vertex, $\operatorname{val}(v)$, will mean the number of edges and legs which meet it. Also, as we consider Riemann surfaces with connected boundary, the leg set will consist of exactly one element. Nevertheless, these methods extend to the multi-component boundary case as well. Stability, the total genus, and the relative homology class of the image curve (i.e., degree and winding) place the usual restrictions on graphs (cf. [23]).

Each graph $\Gamma$ defines a component of the fixed-point locus $M_{\Gamma}=\prod_{v} \bar{M}_{g(v), v a l(v)}$. 
A virtual localization formula à la 15 for open-string Gromov Witten invariants $K_{d, w}^{g}$ would take the form

$$
K_{d, w}^{g}=\int_{\overline{\mathcal{M}}^{v i r}} 1=\sum_{\Gamma} \frac{1}{\left|\mathbf{A}_{\Gamma}\right|} \int_{M_{\Gamma}} \frac{1}{e\left(N_{\Gamma}^{v i r}\right)}
$$

where $e\left(N_{\Gamma}^{v i r}\right)$ represents the Euler class of the virtual normal bundle of $M_{\Gamma}$ inside $\mathcal{M}, i$ is the inclusion map from the fixed-point locus, and $\mathbf{A}_{\Gamma}$ is an automorphism group which is defined as the group $\mathbb{Z} / w \mathbb{Z} \times \mathbf{A}_{\Gamma^{\prime}}$ where $\Gamma^{\prime}$ is the graph with the leg deleted and $\mathbf{A}_{\Gamma^{\prime}}$ is the automorphism group defined in [15]. Concretely, the order of this group, which is all that is relevant here, is $w|\operatorname{Aut}(\Gamma)| \times \prod_{\text {edges }} d_{e}$.

What remains is to calculate the weights of the torus action on the various vector spaces and bundles over the fixed-point loci. The basic exact sequence which describes the "obstruction bundle" calculation is

$$
0 \rightarrow \operatorname{Aut}(C) \rightarrow H^{0}\left(\psi^{*} T M\right) \rightarrow T \mathcal{M} \rightarrow \operatorname{Def}(C) \rightarrow H^{1}\left(\psi^{*} T M\right) \rightarrow O b s \rightarrow 0 .
$$

We interpret this sequence as a sequence of bundles fibered over the moduli

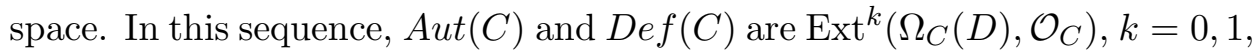
respectively, where $D$ is the divisor of marked points (the nodes, in our example). When $C$ is smooth these spaces are familiar as $H^{0}(T C)$ and $H^{1}(T C)$. The notation $H^{k}\left(\psi^{*} T M\right), k=0,1$, is short for $H^{k}\left(C, \partial C ; \psi^{*} T M, \psi^{*} T L\right)$ over a point $\psi: C \rightarrow M$ in the moduli space, $\mathcal{M}$. For example, when $C$ is a smooth disk, $H^{0}\left(\psi^{*} T M\right)$ represents global holomorphic tangent vectorvalued sections over the disk, which lie in the real sub-bundle $T L$ along the boundary $\partial C \cong S^{1}$ This sequence tells us that

$$
\frac{O b s}{T \mathcal{M}}=\frac{A u t(C)}{\operatorname{Def}(C)} \cdot \frac{H^{1}(T M)}{H^{0}(T M)} .
$$

To compute the right-hand side we use the normalization for $C$ in terms of the irreducible components $C_{i}$ (here $C_{i}$ represents $C_{v}, C_{e}$, and $C_{l}$ ):

$$
\left.0 \rightarrow \mathcal{O}_{C} \rightarrow \bigoplus_{i} \mathcal{O}_{C_{i}} \rightarrow \bigoplus_{n} T M\right|_{\psi\left(x_{n}\right)} \rightarrow 0
$$

where $n$ runs over the nodes $x_{n}$ lying at the intersection of the components. In what follows we will use the related notation of "flags" $F$, with the convention that a flag can lie at the intersection of $C_{v}$ and either an edge or a leg.

We get all the information we need when we tensor Eq. (9) by $\left(\psi^{*} T M, \psi^{*} T L\right)$ : 


$$
\frac{H^{1}(T M)}{H^{0}(T M)}=\frac{\bigoplus_{i} H^{1}\left(C_{i}, \psi^{*} T M\right) \bigoplus_{n} T_{\psi\left(x_{n}\right)} M}{\bigoplus_{i} H^{0}\left(C_{i}, \psi^{*} T M\right)}
$$

The calculation has therefore been reduced to computations of $A u t(C)$, $\operatorname{Def}(C)$, and sections of bundles over the irreducible components of the fixed-loci source curves. Most of this is well-trodden material, and involves keeping track of the torus weights of various line bundles over $\mathbb{P}^{1}$ or twisted Hodge and cotangent line bundles over contracted components. In particular, the $\operatorname{Aut}(C), \operatorname{Def}(C)$, and denominator terms in Eq.(8) which do not involve legs have been discussed in [15]. The other numerator terms not involving legs have been discussed in [22]. The torus weights of legs have been discussed in [21]; we now provide an alternate derivation.

Let $C_{l}$ be a leg component with $\psi$ the map of Eq. (5). We are interested in the vector spaces $H^{k}\left(C_{l}, \partial C_{l} ; \psi^{*} T M, \psi^{*} T L\right), k=0,1$. Let us search for $H^{0}$, the holomorphic sections. (A similar analysis can be applied to $H^{1}$.) Since $C_{l}$ is mapped entirely within the open set $U$ coordinatized by $z, n, f$, the bundle $T M$ is trivial and we can describe holomorphic sections by a triplet $h=\left(h_{1}, h_{2}, h_{3}\right)$ of holomorphic functions of $u$. The boundary condition described by the matrix $A$ in Eq. (6) states that on the boundary,

$$
h\left(e^{i \theta}\right)=A \cdot a,
$$

where $a$ is a triplet of real functions of $\theta$. Since $A$ is block diagonal, we can look at the blocks separately. The northwestern $1 \times 1$ block states $h_{1}\left(e^{i \theta}\right)=a_{1} i e^{i w \theta}$, so $-i e^{-i w \theta} h_{1}$ is a real function. Since $h_{1}$ is holomorphic, we can expand $h_{1}\left(e^{i \theta}\right)=\sum_{k \geq 0} h_{1, k} e^{i k \theta}$, and we then have the equation

$$
-i e^{-i w \theta} \sum_{k=0}^{\infty} h_{1, k} e^{i k \theta}=i e^{i w \theta} \sum_{k=0}^{\infty} \overline{h_{1, k}} e^{-i k \theta},
$$

from which we derive $h_{1, k}=-\overline{h_{1,2 w-k}}, k=0, \ldots, 2 w$, with all others vanishing. The lower block can be treated similarly; one finds that there are no contributions to $H^{0}$, while only the lower block contributes to $H^{1}$. As a result, we find that $H^{0}$ is a real $(2 w+1)$-dimensional vector space, and recalling that $u$ has weight $\lambda_{z} / w$, we see that the action of $S^{1}$ on $H^{0}$ decomposes into representations of the type $\left(\begin{array}{cc}0 & m \\ -m & 0\end{array}\right), m=1, \ldots, w$, and one trivial representation (whose zero weight will cancel against an automorphism). Up to an overall sign, then, we have found that the equivariant 
Chern class of the vector space $H^{0}$ is $\prod_{m=1}^{w}(m / w)\left(\lambda_{3}-\lambda_{1}\right)$, where we have used $\lambda_{z}=\lambda_{1}-\lambda_{3}$. This combination appears in the denominator of the first line of the equation below. As discussed in [10], the orientation on the vector space $H^{k}\left(C_{l}, \psi^{*} T M\right)$ is a subtle issue affecting an overall sign in our calculation. Without a rigorous moduli space to work with, we are unable to determine this sign directly, so we choose a sign convention which leads to agreement with physics and integrality.

Putting these contributions together, one arrives at a formula for the integrand of $\int_{M_{\Gamma}}$ in Eq. (7). In the following, we have written $k(l)$ for the unique fixed point in $\mathbb{P}^{2}$ not equal $i(l)$ or $j(l)$. Also, $\omega_{F} \equiv\left(\lambda_{i(F)}-\lambda_{j(F)}\right) / d$, where $d=d_{e}$ for an edge flag and $d=w(l)$ for a leg flag. $e_{F}$ is the first Chern class of the cotangent line bundle over $M_{\Gamma}$ associated to the point $x_{F} \in C_{v}$. We also write the top Chern class of a twisted dual Hodge bundle as $P_{g}\left(\lambda, E^{*}\right)=\sum_{r=0}^{g} \lambda^{r} c_{g-r}\left(E^{*}\right)$, where $E$ has fibers $H^{0}\left(K_{C_{v}}\right)$. In total, we have the following formula, in which the $\frac{H^{1}}{H^{0}}$ leg contributions are on the first line (for simplicity we have written the formula for general positive windings only), the non-leg $H^{1}\left(K_{\mathbb{P}^{2}}\right)$ contributions on the second line, and the $e\left(N_{\Gamma}^{v i r}\right)^{-1}$ contributions on the following lines (we have performed all genus-zero moduli space integrations, using Kontsevich's formula in [23]):

$$
\begin{aligned}
\frac{i^{*} \phi}{e\left(N^{v i r}\right)}= & \prod_{l} \frac{\prod_{m=1}^{w(l)-1}\left[(-m / w(l))\left(\lambda_{i(l)}-\lambda_{j(l)}\right)-\left(\lambda_{k(l)}-\lambda_{j(l)}\right)\right]}{\prod_{m=1}^{w(l)}(-m / w(l))\left(\lambda_{i(l)}-\lambda_{j(l)}\right)} \\
& \left.\times \prod_{v} \Lambda_{i(v)}^{v a l(v)-1} P_{g(v)}\left(\Lambda_{i(v)}, E^{*}\right) \prod_{e} \prod_{m=1}^{3 d_{e}-1} \Lambda_{i(e)}+\frac{m}{d_{e}}\left(\lambda_{i(e)}-\lambda_{j(e)}\right)\right] \\
& \times \prod_{e} \frac{(-1)^{d_{e}} d_{e}^{2 d_{e}}}{\left(d_{e} !\right)^{2}\left(\lambda_{i(e)}-\lambda_{j(e)}\right)^{2 d_{e}}} \prod_{\substack{a+b=d_{e} \\
a, b>0 \\
k \neq i(e), j(e)}} \frac{1}{\frac{a}{d_{e}} \lambda_{i(e)}+\frac{b}{d_{e}} \lambda_{j(e)}-\lambda_{k}} \\
& \times \prod_{v} \prod_{j \neq i(v)}\left(\lambda_{i(v)}-\lambda_{j}\right)^{v a l(v)-1} \\
& \times\left\{\prod _ { v } \left[\left(\sum_{F} w_{F}^{-1}\right)^{v a l(v)-3} \quad \text { if } g(v)=0,\right.\right. \\
\prod_{v} \prod_{j \neq i(v)} P_{g(v)}\left(\lambda_{i(v)}-\lambda_{j}, E^{*}\right) \prod_{F \ni v} \frac{1}{w_{F}-e_{F}} & \text { if } g(v) \geq 1,
\end{aligned}
$$

where $\Lambda_{i} \equiv \lambda_{1}+\lambda_{2}+\lambda_{3}-3 \lambda_{i}$. As a small check, one can see that the $\Lambda_{i}^{-1}$ term of the second line and the $\left(\lambda_{i(v)}-\lambda_{j(v)}\right)^{-1}$ terms of the fourth line come from the node contributions to $H^{1}\left(\psi^{*} T M\right)$ in the numerator of Eq.(10). 
These formulas can be generalized for Lagrangians in other noncompact, toric Calabi-Yau geometries as well.

\section{Mirror Symmetry for Open String Invariants}

The techniques of the previous section apply in all genera, but in genus zero more can be said. Here we show how a simple observation, together with the equivariant mirror theorem, leads to a kind of "proof" of mirror symmetry in genus zero.

We return to the geometry of Section 2. We will consider open-string Gromov-Witten invariants for stable maps with a single boundary component, and with a positive winding, so that the leg at the fixed locus is forced to attach at the "north pole," $p(z=n=f=0)$. As we now show, an inspection of the contribution of the legs in Eq. (10) shows that the calculation of the Gromov-Witten invariant differs only by a winding-dependent leg factor from a one-pointed stable map calculation, where the marked point is forced to map to the north pole. The reason is simple. The fixed loci are described by the same data: knowing where the marked point is on the source curve tells you where to attach a leg with winding $w$. An overall factor will account for the contribution of the leg to Eq. (10). What remains is the fact that the tangent to the source curve at the attached point affects $\operatorname{Def}(C)$. We therefore write down a one-pointed Gromov-Witten invariant which is tailor-made to contribute the appropriate factor in $\operatorname{Def}(C)$ at the marked point.

Let $K_{d, w} \equiv K_{d, w}^{0}$ be the open-string Gromov-Witten invariant of Eq. (7) in genus zero at degree $d$ with winding $w>0$. Recall that $K_{d, w}$ has only a calculational definition. Using this definition, it is simple to show that

$$
K_{d, w}=\mathcal{C}_{w} \int_{\overline{\mathcal{M}}_{0,1}\left(\mathbb{P}^{2}, d\right)} e\left(E_{d}\right) \cdot \frac{e v^{*}\left(\phi_{p}\right)}{\lambda-\psi}
$$

where $e\left(E_{d}\right)$ is the equivariant Euler class of the obstruction bundle $E_{d} \equiv$ $R^{1} \rho_{*} e v^{*} K_{\mathbb{P}^{2}} ; \phi_{p}$ is the equivariant class of the north pole; $e v^{*}$ is the pull-back under the evaluation map ev $: \overline{\mathcal{M}}_{0,1}\left(\mathbb{P}^{2}, d\right) \rightarrow \mathbb{P}^{2}$; and

$$
\lambda \equiv-\lambda_{z} / w
$$

is the weight of the tangent space of the leg at the point of attachment. $\mathcal{C}_{w}$ is the overall factor discussed above, and is defined by

$$
\mathcal{C}_{w} \equiv \frac{1}{\left(-\lambda_{n}\right)\left(-\lambda_{z}\right)} \cdot C_{w}, \quad \text { where } \quad C_{w} \equiv \frac{\lambda_{n}+\lambda_{z}}{-w \lambda_{z}} \prod_{k=1}^{w-1} \frac{\frac{k}{w} \lambda_{z}+\lambda_{n}}{\frac{k}{w} \lambda_{z}}
$$


(the factor of $w$ in the denominator accounts for automorphisms of the leg). In the above, $\int \overline{\mathcal{M}}_{0,1}\left(\mathbb{P}^{2}, d\right)$ represents the equivariant push-forward under the evaluation map, and will henceforth be written $e v_{*}$. Noting that $i_{p}^{*}\left(\phi_{p}\right)$ has the effect of multiplying by $\left(-\lambda_{z}\right)\left(-\lambda_{n}\right)$, we can cancel out these terms and write

$$
K_{d, w}=C_{w} \cdot i_{p}^{*} e v_{*}\left(\frac{e\left(E_{d}\right)}{\lambda-\psi}\right)
$$

Note that here we are interpreting $K_{d, w}$ as an element of $\mathbb{Q}\left(\lambda_{1}, \lambda_{2}, \lambda_{3}\right)$ since our prescription for computing it yields not a number, but a rational function of the weights of the torus. Similarly, the other side of this equation is an element of the localized equivariant cohomology of a point, so the above equation makes sense.

\subsection{Equivariant Mirror Theorem}

The mirror theorems of Givental [11] [12] and Lian-Liu-Yau [26] are actually proven in the equivariant setting. Using their results we have an algebraic procedure for calculating $e v_{*}\left(\frac{e\left(E_{d}\right)}{\lambda-\psi}\right)$, hence $K_{d, w}$, which we now describe. (The theorem does not apply at higher genus, where the explicit graph sum is the only approach currently available.)

Let $H$ be the equivariant hyperplane class on $\mathbb{P}^{2}$ (so $H$ restricted to the $i$ th fixed point is $\lambda_{i}$ ). Let $K$ be the equivariant first Chern class of the canonical bundle with its canonical torus action. Let us define $J$ and $\widetilde{J}$ by

$$
J \equiv e^{\frac{t_{0}+H t_{1}}{x}} \widetilde{J} \equiv e^{\frac{t_{0}+H t_{1}}{x}}\left(1+K \cdot \sum_{d>0} q^{d} e v_{*} \frac{e\left(E_{d}\right)}{x(x-\psi)}\right)
$$

as well as $I$ and $\widetilde{I}$ by

$$
I \equiv e^{\frac{t_{0}+H t_{1}}{x}} \widetilde{I} \equiv e^{\frac{t_{0}+H t_{1}}{x}} \sum_{d \geq 0} q^{d} \frac{\prod_{m=0}^{3 d-1}(K-m x)}{\prod_{m=1}^{d} \prod_{i=1}^{3}\left(H-\lambda_{i}+m x\right)},
$$

where $q=e^{t_{1}}$. Finally, define $I_{1}$ by

$$
I=e^{\frac{t_{0}+H t_{1}}{x}}\left(1+I_{1} \frac{H}{x}+o\left(\frac{1}{x^{2}}\right)\right) .
$$

Explicity,

$$
I_{1}=\frac{K}{H} \sum_{d=1}^{\infty} \frac{(-1)^{d+1}(3 d-1) !}{(d !)^{3}} q^{d}
$$


Then the equivariant mirror theorem states that

$$
J\left(t_{0}, t_{1}+I_{1}\right)=I\left(t_{0}, t_{1}\right) .
$$

(See [8] or [26] for this formula, but note that in their calculations, a different linearization on $K_{\mathbb{P}^{2}}$ is used.) In implementing this change of variables, one must not neglect that $q=e^{t_{1}}$ should be replaced by $e^{t_{1}+I_{1}}=q e^{I_{1}(q)}$ on the left side. To make things more concrete, we can substitute $\lambda=-\lambda_{z} / w$ for $x$ and replace equivariant classes by their restrictions via $i_{p}^{*}$. Then $H=\lambda_{3}$, $K=\lambda_{1}+\lambda_{2}-2 \lambda_{3}$, and as always $\lambda_{z}=\lambda_{1}-\lambda_{3}$ and $\lambda_{n}=\lambda_{2}-\lambda_{3}$. Now defining $Q(q)$ by

$$
Q(q)=q e^{I_{1}(q)},
$$

one can write Eq. (16) as $J(Q)=I(q)$. In practice, it is useful to invert and solve for $q(Q)$ (see Eq.(24)) to the order needed in calculations.

This procedure yields the same $K_{d, w}$ as the graph sum.

\subsection{Mirror Symmetry}

Now that we have a power-series prescription for computing the open-string Gromov-Witten invariants - defined by the graph sum and expressible via Eq. (13) in terms of equivariant invariants of one-pointed maps - we can ask if this procedure yields the same numbers that we get from physics via the B-model and mirror symmetry [1] [2].

Let us review the physics approach. First form the generating function

$$
W(Q, y)=\sum_{d, w} K_{d, w} Q^{d} y^{w}
$$

where $Q=e^{-\hat{t}}$ is the Kähler parameter and $y=e^{\hat{u}}$ is the complexified holonomy parameter. We have

$$
W=\int \hat{v}(\hat{u}) d \hat{u}, \quad \text { or } \quad \partial_{\hat{u}} W=\hat{v}(\hat{u}),
$$

where $\hat{v}$ and $\hat{u}$ are classically equal to the un-hatted parameters obeying the following equation:

$$
e^{u}+e^{v}+1+e^{-t-u-v}=0
$$

or

$$
v=\log \left[-\left(1+e^{u}\right) / 2-\frac{1}{2} \sqrt{\left(1+e^{u}\right)^{2}-4 e^{-t} e^{-u}}\right] .
$$


[The origin of the equation is a specialization of $X_{1}+X_{2}+X_{3}+X_{4}=0$ in the patch $X_{4}=1$ subject to the constraint $X_{1} X_{2} X_{3} X_{4}^{-3}=e^{-t}$. Here we have written $X_{1}=e^{u}, X_{2}=e^{v}, X_{3}=e^{-t-u-v}$. Note that the exponents of this constraint, $(1,1,1,-3)$, are precisely the toric data for $K_{\mathbb{P}^{2}}$.]

In fact, the relation of the parameters in Eq. (19) and their hatted cousins receives instanton corrections. More precisely, $u$ and $v$, the parameters in the complex equation, are not the "flat coordinates." The map from the parameters in the equation to the flat Kähler parameters is what we need to perform enumerative tests. We have $\hat{u}=\hat{u}(u, t)$ and $\hat{v}=\hat{v}(v, t)$. The relation between the Kähler parameter $\hat{t}$ and the complex parameter $z=e^{-t}$ is well known: $\hat{t}$ is the logarithmic solution to the Picard-Fuchs equation for the Fermat cubic in $\mathbb{P}^{2}$ ). The other relations were found in [2]. Explicitly, for our computation, we have

$$
\begin{aligned}
\hat{u}(u, t) & =u+(t-\hat{t}) / 3+i \pi \quad \text { or } \quad e^{\hat{u}}=-e^{u} e^{\Delta / 3} \\
\hat{v}(v, t) & =v+(t-\hat{t}) / 3+i \pi \quad \text { or } \quad e^{\hat{v}}=-e^{v} e^{\Delta / 3} \\
\Delta & \equiv t-\hat{t}=\sum_{k=1}^{\infty}=\frac{(-1)^{k}}{k} \frac{(3 k) !}{(k !)^{3}} z^{k}
\end{aligned}
$$

where again $z=e^{-t}$. The Picard-Fuchs equation is $\mathcal{L} f=0$, where $\mathcal{L}=$ $\theta^{3}+3 z(3 \theta+1)(3 \theta+2)$ and $\theta \equiv z \frac{d}{d z}$.

Now if we use Eq. (20) with Eqs. (21)-(23), we can solve for $\hat{v}$ in terms of $\hat{u}$. First, following [2], we define $r \equiv e^{\Delta / 3}=\left(\frac{Q}{z}\right)^{\frac{1}{3}}=1-2 Q+5 Q^{2}-32 Q^{3}+\ldots$, where we have used the inverse relation

$$
z(Q)=Q+6 Q^{2}+9 Q^{3}+56 Q^{4}-\ldots
$$

$(Q(z)$ is obtaind from Eq. (23) by exponentiation). We get

$$
\hat{v}=\log \left[(r-y) / 2+\sqrt{(r-y)^{2} / 4+Q / y}\right] .
$$

Then Eq. (17) and $\hat{v}=\partial_{\hat{u}} W$ tell us

$$
\hat{v}=\sum_{d, w} w K_{d, w} Q^{d} y^{w} .
$$

From Eqs. (25) and (26) we can read off the Gromov-Witten invariants, $K_{d, w}$. [?

\footnotetext{
${ }^{3}$ To connect with the notation of the previous section, set $t=-t_{1}$, so $z=q$. Thus the inverse relation $z(Q)$ given in Eq. (24) is the same one needed for the equivariant mirror theorem.
} 


\subsection{Equivalence}

We now show that the two methods of computing open-string invariants are the same. We are grateful to A. Klemm for helping us with this verification.

To make this explicit, we will use a specific set of weights, namely $\lambda_{z}=1$, $\lambda_{n}=0$ (this corresponds to "ambiguity zero" in the physics). In this case, $K=1$ and $H=0$, as explained below Eq. (16), while $C_{w}=-1 / w$.

Note $\widetilde{J}(Q, x=-1 / w)=1+\sum_{d>0} Q^{d} w^{2} K_{d, w}$. Here it is convenient to note $K_{0, w}=1 / w^{2}$, which is the pure leg contribution. Then

$$
\sum_{w \neq 0} \widetilde{J}(Q,-1 / w) y^{w}=\sum_{d \geq 0 ; w \neq 0} w^{2} K_{d, w}
$$

Comparing with Eq. (26), we see that this is $\partial_{\hat{u}} \hat{v}$, or equivalently $\partial_{\hat{u}}^{2} W(Q, y)$ (recall $y=e^{\hat{u}}$. Now let's take Eq. (25) and find $\partial_{\hat{u}} \hat{v}$. Some algebra leads to

$$
\partial_{\hat{u}} \hat{v}=-\frac{1}{2}-\frac{r-3 y}{4 \sqrt{(r-y)^{2} / 4+Q / y}},
$$

On the other hand, the mirror theorem of Eq. (16) together with Eq. (15) with our choice of weights gives

$$
\sum_{w \neq 0} \widetilde{J}(Q,-1 / w) y^{w}=\sum_{w \neq 0, d \geq 0} y^{w} r^{-w} q^{d}(-1)^{d} w \frac{(w+3 d-1) !}{(d !)^{2}(w+d) !} .
$$

Thus we predict that, up to constants, we have the following equivalence of power series:f

$$
-\frac{r-3 y}{4 \sqrt{(r-y)^{2} / 4+Q / y}}=\sum_{\substack{w \neq 0 \\ d \geq 0}} y^{w} r^{-w} q^{d}(-1)^{d} w \frac{(w+3 d-1) !}{(d !)^{2}(w+d) !},
$$

with $q(Q)$ and $r(Q)$ given by Eq. (24) and $r=(Q / q)^{1 / 3}$. To prove this, we define $a=y / r$ and note that the left hand side is equal to

$$
\frac{3 a-1}{2(1-a)}\left(1+\frac{4 q}{a(1-a)^{2}}\right)^{-1 / 2}=\frac{3 a-1}{2} \sum_{d=0}^{\infty} \frac{(2 d-1) ! !}{(2 d) ! !}(-1)^{d} \frac{4^{d} q^{d}}{a^{d}(1-a)^{2 d+1}},
$$

where we have used $(1+x)^{-1 / 2}=\sum_{n=0}^{\infty} \frac{(2 n-1) ! !}{(2 n) ! !}(-1)^{n} x^{n}$, with $(2 n) ! ! \equiv$ $2^{n}(n !)$. Now expanding $(1-a)^{-k}=\sum_{n=0}^{\infty} \frac{(k+n-1) !}{n !(k-1) !} a^{n}$, the coefficient of $a^{m-d} q^{d}$ is seen to be

$$
\frac{(-4)^{d}(2 d-1) ! !}{2 \cdot(2 d) ! !}\left[\frac{3(2 d+m-1) !}{(2 d) !(m-1) !}-\frac{(2 d+m) !}{(2 d) ! m !}\right]=(-1)^{d}(m-d) \frac{(m+2 d-1) !}{(d !)^{2} m !} .
$$

\footnotetext{
${ }^{4}$ Equality holds if we add $1 / 2$ to the left hand side
} 
The same coefficient appears on the right hand side, with $w=m-d$. Curiously, the explicit form of $Q(q)$ is not used.

For nonzero ambiguity $s$, one makes the change of $y \rightarrow y e^{s \hat{v}}$ in Eq. (25) and re-solves for $\hat{v}$ before performing the check, where now $C_{w}$ of Eq. (12) is taken with $\lambda_{z}=1, \lambda_{n}=s$.

\section{Calculations}

We have implemented a computer program running in Maple which fully automates the calculation described in Section 3. A similar program for graphs without legs was described in [22]. The program computes openstring Gromov-Witten invariants, as listed in Table 1.

Let us first display some calculations in "ambiguity zero." The ambiguity $p$ encountered in physical calculations corresponds to $\lambda_{z}=1$ and $\lambda_{n}=p$. (When $\lambda_{z} \neq 1$ the calculations do not lead to integer invariants.)

\begin{tabular}{|lrr|}
\hline$g$ & $d=1$ & $d=2$ \\
\hline 0 & -1 & $\frac{1}{4}\left(w^{2}+4 w+15\right)$ \\
1 & $\frac{1}{24}\left(w^{2}-2\right)$ & \\
2 & $-\frac{1}{5760}\left(3 w^{4}-20 w^{2}+24\right)$ & $\frac{w}{23040}\left(39 w^{5}+364 w^{4}+1185 w^{3}+1200 w^{2}-632 w-1056\right)$ \\
\hline
\end{tabular}

Table 1: Some open-string Gromov-Witten invariants $K_{d, w}^{g}$ for general (positive) winding, $w$ (with ambiguity $p=0$ ).

A surprising result of these calculations is the weight dependence of the results. This is in contrast to the usual, non-equivariant calculations of Gromov-Witten invariants. Physically, this ambiguity is related to different possible "special coordinates" in the mirror B-model, and to the framing of a link in a Chern-Simons theory [2] (the Chern-Simons calculation is only relevant to the $\mathcal{O}_{\mathbb{P}^{1}}(-1,-1)$ example of [21] [25]).

At genus zero, our results agree with the predictions of Aganagic, Klemm, and Vafa, including the dependence on the torus weights, i.e. on the "ambiguity" $p=\lambda_{n}$. Tables appear in their paper [2].

At degree zero, for the genera computed $(g \leq 4)$, our results agree with the predictions of Ooguri and Vafa 29] and the computations of Katz and Liu [21] and Li and Song [25]. Namely, at ambiguity zero, degree zero, winding $w$ and genus $g$ the Gromov-Witten invariant $K_{0, w}^{g}(p=0)$ is given as the negative of the $u^{2 g-2+1}$ term in the expansion of $(1 / w)[2 \sin (w u / 2)]^{-1}$. 
For example, $K_{0,3}^{4}=-3429 / 5734400$.

At higher genera and degree, there are no known predictions from physics of what the higher genus open-string Gromov-Witten formulas should be, though the work of Ooguri and Vafa [29] and of Labastida, Mariño and Vafa [24] leads to strong integrality checks, which hold for the invariants we compute. Let $n_{d, w}^{g}$ denote the integer invariants representing the number of BPS domain walls. To demonstrate integrality, consider the invariants $K_{2,3}^{g}$, i.e. the right column of the Table 1 , with $w=3$. Since $d$ and $w$ are relatively prime, there are only contributions to this term from $n_{d, w}^{g}$ with $(d, w)=(2,3)$. Using the integrality relations of [24] (Eq. (2.11) of [27), we find from Table $1, K_{2,3}^{0}=-n_{2,3}^{0}=9$, so $n_{2,3}^{0}=-9$. Then $K_{2,3}^{1}=$ $(7 / 24) n_{2,3}^{0}+n_{2,3}^{1}=-109 / 8$, so $n_{2,3}^{1}=-11$. Finally, $K_{2,3}^{2}=(-29 / 1920) n_{2,3}^{0}+$ $(-3 / 8) n_{2,3}^{1}-n_{2,3}^{2}=6567 / 640$, and we find $n_{2,3}^{2}=-6$. If instead we consider $w=2$, then there are more contributions to the Gromov-Witten invariant due to multiple windings. The integer invariants are listed in Table 2.

\begin{tabular}{|rrr|}
\hline$g$ & $d=1$ & $d=2$ \\
\hline 0 & 1 & $-\frac{1}{4}\left(w^{2}+4 w+16-\epsilon_{w}\right)$ \\
1 & 0 & $-\frac{1}{48}\left(w^{4}+8 w^{3}+20 w^{2}+16 w+3 \epsilon_{w}\right)$ \\
2 & 0 & $-\frac{1}{2880}\left(2 w^{6}+24 w^{5}+95 w^{4}+120 w^{3}-52 w^{2}-144 w-45 \epsilon_{w}\right)$ \\
\hline
\end{tabular}

Table 2: Some integer invariants $n_{d, w}^{g}$ for various general (positive) winding, $w$ (with ambiguity $p=0$ ). Here $\epsilon_{w}=\left(1-(-1)^{w}\right) / 2$.

It is also easy to compute the open-string Gromov-Witten invariants corresponding to multiple boundary components ("holes"). Once again, the integrality tests have held up in each calculation checked. For example, we find for a surface with two boundary components with windings $\vec{n}=(1, w)$ that $n_{d=2, \vec{n}=(1, w)}^{g=1}=(-1 / 24)\left(w^{4}+10 w^{3}+35 w^{2}+50 w+48\right)$, an integer.

Of course, it would be extremely desirable to develop recursive differential equations for topological partition functions which would be an openstring version of the BCOV equations [4. The lack of $(2,2)$ worldsheet symmetry will complicate matters significantly. Perhaps the calculations of this paper can play some role in the establishing such equations. 5 or in resolving an open-string version of the holomorphic ambiguity as analogous calculations do in the closed string case 22].

\footnotetext{
${ }^{5}$ Two recent papers have taken steps towards doings so 14
} 


\section{Acknowledgments}

Thanks to M. Aganagic, A. Klemm, and C. Vafa for many discussions of their work; to E. Getzler; to J. Bryan; and to R. Pandharipande and A. Klemm for their help with Section 4 . The work of E.Z. is supported in part by NSF Grant DMS-0072504 and an Alfred P. Sloan Foundation Fellowship. T.G. is partially supported by an NSF postdoctoral fellowship.

\section{References}

[1] M. Aganagic and C. Vafa, "Mirror Symmetry, D-Branes and Counting Holomorphic Discs," hep-h/0105045.

[2] M. Aganagic, A. Klemm, and C. Vafa, "Disk Instantons, Mirror Symmetry and the Duality Web," hep-th/0105045.

[3] Batyrev, Victor V.(D-ESSN) Dual polyhedra and mirror symmetry for Calabi-Yau hypersurfaces in toric varieties. J. Algebraic Geom. 3 (1994) 493-535.

[4] M. Bershadsky, S. Cecotti, H. Ooguri, and C. Vafa, "Holomorphic Anomalies in Topological Field Theories," Nucl. Phys. 405B (1993) 279-304; M. Bershadsky, S. Cecotti, H. Ooguri, and C. Vafa, "KodairaSpencer Theory of Gravity and Exact Results for Quantum String Amplitudes," Commun. Math. Phys. 165 (1994) 311-428.

[5] J. Bryan and R. Pandharipande, "BPS states of curves in Calabi-Yau 3-folds," math.AG/0009025; Geom. Topol. 5 (2001) 287-318.

[6] P. Candelas, X. C. De La Ossa, P. Green, and L. Parkes, "A Pair of Calabi-Yau Manifolds as an Exactly Soluble Superconformal Theory," Nucl. Phys. B359 (1991) 21.

[7] T.-M. Chiang, A. Klemm, S.-T. Yau, and E. Zaslow, "Local Mirror Symmetry: Calculations and Interpretations," hep-th/9903053; Advances in Theoretical and Mathematical Physics 3 (1999).

[8] A. Elezi, "Mirror Symmetry for Concavex Vector Bundles on Projective Spaces," math.AG/0004157.

[9] C. Faber, "Algorithms for Computing Intersection Numbers on Moduli Spaces of Curves, with an Application to the Class of the Locus of Jacobians," math.AG/9706006; in New Trends in Algebraic Geometry: 
EuroConference in Algebraic Geometry, Warwick, 1996, K. Hulek, ed., London Math. Soc. Lecture Note Ser. 264, Cambridge Univ. Press (New York) 1999.

[10] K. Fukaya, Y.-G. Oh, H. Ohta, and K. Ono, "Lagrangian Intersection Floer Theory - Anomaly and Obstruction," preprint Kyoto-Math 0017.

[11] A. Givental, "Equivariant Gromov-Witten invariants," Int. Math. Res. Notices 13 (1996), 613-663.

[12] A. Givental, "A mirror theorem for toric complete intersections," math.AG/9701016.

[13] R. Gopakumar and C. Vafa, "M-theory and Topological Strings-I \& II," hep-th/9809187, hep-th/9812127.

[14] S. Govindarajan, T. Jayaraman, and T. Sarkar, "Disc Instantons in Linear Sigma Models," hep-th/0108234.

[15] T. Graber and R. Pandharipande, "Localization of Virtual Classes," Invent. Math. 135 (1999) 487-518.

[16] K. Hori, "Linear Models of Supersymmetric D-Branes," hepth/0012179.

[17] S. Hosono, M.-H. Saito, and A. Takahashi, "Holomorphic Anomaly Equation and BPS State Counting of Rational Elliptic Surface," hepth/9901151.

[18] S. Hosono, M.-H. Saito, and A. Takahashi, "Relative Lefschetz Action and BPS State Counting," math.AG/0105148.

[19] S. Hosono, A. Klemm, S. Theisen, and S.-T. Yau, "Mirror Symmetry, Mirror Map and Applications to Complete Intersection Calabi-Yau Spaces," Nucl. Phys. B433 (1995) 501-554.

[20] S. Katz, A. Klemm, and C. Vafa, "M-Theory, Topological Strings and Spinning Black Holes," hep-th/9910181.

[21] S. Katz and C.-C. Liu, "Enumerative Geometry of Stable Maps with Lagrangian Boundary Conditions and Multiple Covers of the Disc," math.AG/0103074. 
[22] A. Klemm and E. Zaslow, "Local Mirror Symmetry at Higher Genus," to appear in the Proceedings of the 1999 Winter School on Mirror Symmetry and Special Lagrangians; hep-th/9906046.

[23] M. Kontsevich, "Enumeration of Rational Curves via Torus Actions," in The Moduli Space of Curves, Dijkgraaf et al eds., Progress in Mathematics 129, Birkhäuser (Boston) 1995.

[24] J. Labastida, M. Mariño, and C. Vafa. "Knots, Links and Branes at Large $N$," hep-th/0001102.

[25] J. Li and Y. Song, "Open String Instantons and Relative Stable Morphisms," hep-th/0103100.

[26] B. Lian, K. Liu, and S.-T. Yau, "Mirror Principle I," Asian J. Math. Vol. 1, no. 4 (1997), 729-763.

[27] M. Mariño and C. Vafa, "Framed Knots at Large N," hep-th/0108064.

[28] P. Mayr, "N=1 Mirror Symmetry and Open/Closed String Duality," hep-th/0108229.

[29] H. Ooguri and C. Vafa, "Knot Invariants and Topological Strings," hep-th/9912123; Nucl. Phys. B577 (2000) 419-438.

[30] E. Witten, "Chern-Simons Gauge Theory as a String Theory," hepth/9912123; in The Floer Memorial Volume, H. Hofer et al, eds., Progr. Math. 133 (Birkhäuser, 1995) 637-678. 